\title{
Interictal scalp fast oscillations as a marker of the seizure onset zone
}
L.P. Andrade-Valenca, $\mathrm{MD}, \mathrm{PhD}$
F. Dubeau, MD
F. Mari, $\mathrm{MD}, \mathrm{PhD}$
R. Zelmann, MEng
J. Gotman, PhD

Address correspondence and reprint requests to Dr. AndradeValenca, 3801 University Street, Montreal Neurological Institute, McGill University, Montreal, Quebec, Canada H3A 2B4 lupatrizia@yahoo.com.br

\section{Editorial, page 518}

Supplemental data at www.neurology.org

Supplemental Data

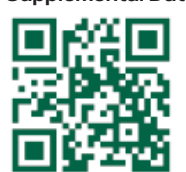

\section{ABSTRACT}

Objective: This study aims to identify if oscillations at frequencies higher than the traditional EEG can be recorded on the scalp EEG of patients with focal epilepsy and to analyze the association of these oscillations with interictal discharges and the seizure onset zone (SOZ).

Methods: The scalp EEG of 15 patients with focal epilepsy was studied. We analyzed the rates of gamma $(40-80 \mathrm{~Hz})$ and ripple $(>80 \mathrm{~Hz})$ oscillations, their co-occurrence with spikes, the number of channels with fast oscillations inside and outside the SOZ, and the specificity, sensitivity, and accuracy of gamma, ripples, and spikes to determine the SOZ.

Results: Gamma and ripples frequently co-occurred with spikes ( $77.5 \%$ and $63 \%$ of cases). For all events, the proportion of channels with events was consistently higher inside than outside the SOZ: spikes (100\% vs $70 \%)$, gamma (82\% vs 33\%), and ripples (48\% vs $11 \%) ; p<0.0001$. The mean rates (events $/ \mathrm{min}$ ) were higher inside than outside the SOZ: spikes (2.64 \pm 1.70 vs $0.69 \pm 0.26, p=0.02)$, gamma (0.77 \pm 0.71 vs $0.20 \pm 0.25, p=0.02)$, and ripples $(0.08 \pm$ 0.12 vs $0.04 \pm 0.09, p=0.04)$. The sensitivity to identify the SOZ was spikes $100 \%$, gamma $82 \%$, and ripples $48 \%$; the specificity was spikes $30 \%$, gamma $68 \%$, and ripples $89 \%$; and the accuracy was spikes $43 \%$, gamma $70 \%$, and ripples $81 \%$.

Conclusion: The rates and the proportion of channels with gamma and ripple fast oscillations are higher inside the SOZ, indicating that they can be used as interictal scalp EEG markers for the SOZ. These fast oscillations are less sensitive but much more specific and accurate than spikes to delineate the SOZ. Neurology ${ }^{\circledR} 2011 ; 77: 524-531$

\section{GLOSSARY}

$\mathbf{F I R}=$ finite-impulse response; HFO = high-frequency oscillation; $\mathbf{M N I}=$ Montreal Neurological Institute; $\mathbf{S O Z}=$ seizure onset zone.

The conventional range of EEG analysis usually involves frequencies below $40 \mathrm{~Hz}$. Studies over the last decade suggest, however, that high-frequency oscillations (HFOs) may have an essential role in normal and pathologic brain function. Reports using microelectrodes combined with depth EEG electrodes showed the presence of HFOs in epileptic patients. ${ }^{1-3}$ HFOs were also found using standard macroelectrodes during the ictal ${ }^{4}$ and interictal periods..$^{5-8}$ They appear to be a good indicator of the seizure onset zone (SOZ) and of disease activity ${ }^{9}$ and possibly predict outcome after epilepsy surgery. ${ }^{10}$

For clinical application, it would be preferable to record fast oscillations noninvasively. Early studies on scalp EEG identified fast oscillations in only $0.2 \%-3.4 \%$ of epileptic patients. ${ }^{11-13}$ Recently, there has been a renewed interest for the study of fast oscillations with scalp EEG. ${ }^{14-19}$ While infrequent, interictal fast oscillations were specific in identifying children with epilepsy and localized ictal onsets. ${ }^{16}$ Ictal gamma activity was recorded on scalp EEG during epileptic spasms and differed from muscle artifacts. ${ }^{18,19}$ Even frequencies in the ripple band were measured in children with status epilepticus during sleep. ${ }^{14}$

From the Montreal Neurological Institute (L.P.A.-V., F.D., F.M., R.Z., J.G.), McGill University, Montreal, Canada; and Department of Neurology (L.P.A.-V.), University of Pernambuco-UPE, Recife, Pernambuco, Brazil.

Study funding: Supported by the Canadian Institutes of Health Research grants MOP-102710, MOP 10189.

Disclosure: Author disclosures are provided at the end of the article. 
This study aims to identify interictal fast oscillations in the gamma $(40-80 \mathrm{~Hz})$ and in the ripple $(>80 \mathrm{~Hz})$ bands on the scalp EEG of epileptic patients and to characterize the relationship between these oscillations and the interictal epileptiform discharges and the SOZ. We hypothesize that interictal fast oscillations can be recorded on the scalp EEG and could be markers of epileptogenic brain tissue.

METHODS Patient selection and recording methods. Fifteen consecutive patients ( $31.7 \pm 11.6$ years old, 13 women) with focal epilepsy were enrolled and underwent EEG-telemetry investigation at the Montreal Neurological Institute (MNI). The

Figure 1 Patient 10: Examples of artifacts and ripple oscillations
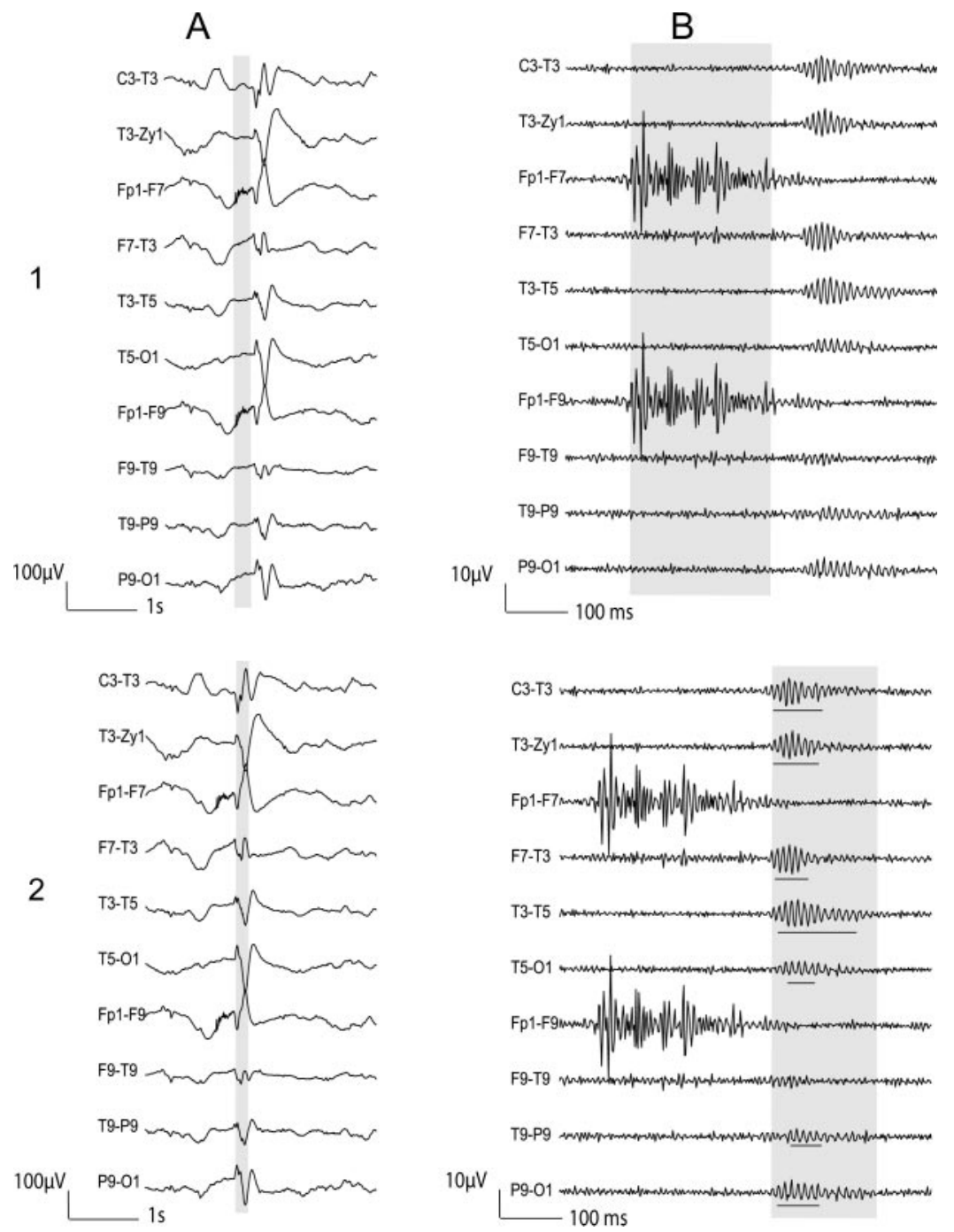

1) Short EMG bursts. 2) Ripples co-occurring with sharp wave. (A) Raw EEG. (B) EEG filtered with high-pass filter of $80 \mathrm{~Hz}$. Gray section in $\mathrm{A}$ is expanded in time and amplitude in $\mathrm{B}$. Note that for this and subsequent figures the calibration is different in the left and right part of the figure, but is the same for the top and bottom parts. Ripple oscillations are underlined. The waveform morphology of nonartifactual fast oscillations is more rhythmic and regular in amplitude and frequency than artifactual oscillations. clinical purposes of EEG-telemetry included determination of seizure classification and epilepsy syndrome, and presurgical evaluation. The only inclusion criterion was the presence of at least one spike per minute during a previous record.

Recordings were performed using the Harmonie system (Stellate, Montreal, Canada) with scalp electrodes placed according to the international $10-20$ system, with additional zygomatic electrodes and electrodes at F9/F10, T9/T10, and P9/P10, and reference $\mathrm{CPz}$. EEG acquisition comprised a low pass filter at $200 \mathrm{~Hz}$ and sampling at $600 \mathrm{~Hz}$.

Standard protocol approval, registrations, and patient consents. This study was approved by the MNI and Hospital Research Ethics Committee and all patients signed an informed consent.

Data selection and marking of spikes and fast oscillations. We started with 457 channels (14 patients with 31 channels and one patient with 23 channels). We excluded 14 channels because of malfunction or continuous artifacts, leaving 443 channels for analysis. Analyses were performed using a bipolar montage, during periods of 30 minutes of non-REM sleep identified visually. Sleep records were chosen since fast oscillations and spikes occur most frequently in this period..$^{20,21}$

The seizure onset zone (SOZ) was defined as the scalp area with the first ictal discharge prior to or concomitant with clinical onset. All channels involved at the beginning of the ictal electrographic discharge were considered as the SOZ. If the clinical onset preceded the EEG onset, the onset was considered as nonlocalized. Spikes and fast oscillations were visually marked independently of each other. Spikes were marked using a $10 \mathrm{~s} /$ page time scale. The spike markers were then made invisible so that the marking of fast oscillations was not biased by the knowledge of spike localization. We considered fast oscillations only events containing at least 4 consecutive oscillations and amplitude clearly greater than the background. For identifying fast oscillations, channels were displayed with a $250 \mathrm{~mm} / \mathrm{s}$ time scale. The computer screen was split to show the scalp EEG with the high-pass filter at 40 $\mathrm{Hz}$ and at $80 \mathrm{~Hz}$ simultaneously, thus removing the "standard" EEG and showing only fast activity. A finite-impulse response (FIR) filter was used to minimize ringing. We categorized fast oscillations as gamma activity $(40-80 \mathrm{~Hz})$ and ripples $(>80 \mathrm{~Hz})$. This procedure is the same as that used in our studies of intracerebral HFOs. ${ }^{7,9}$ After marking all events, the EEG was reviewed a second time by the same reviewer for verification.

It is possible that HFOs are artifactual, particularly coming from the EMG activity from scalp muscles, since this activity includes the frequencies of interest in this study. This possibility is diminished but not eliminated by the selection of sleep periods. We developed a procedure to identify oscillations of noncerebral origin. The procedure and examples are described in Results.

Data analysis. We calculated for each channel rates of spike, gamma, and ripple oscillations per minute (computed for every 1-minute interval) and the co-occurrence of spikes and fast oscillations; co-occurrence was an overlap of the 2 markers, independently of the duration or sequence of both events. All the channels studied, with or without fast oscillations or spikes, were classified as inside or outside the SOZ.

We analyzed the rates of gamma and ripple, co-occurrence of spikes and fast oscillations, number of channels with fast oscillations inside and outside the $\mathrm{SOZ}$, as well as the specificity, sensitivity, and accuracy of gamma, ripples, and spikes to determine the SOZ. 
Sensitivity was defined as [SOZ channels with fast oscillations/(SOZ channels with fast oscillations + SOZ channels without fast oscillations) $\times 100$, specificity as [non-SOZ channels without fast oscillations/(non-SOZ channels without fast oscillations + non-SOZ channels with fast oscillations) $] \times 100$, and accuracy [(SOZ channels with fast oscillations + non-SOZ channels without fast oscillations)/total channels] $\times 100$.

We applied the Kolmogorov-Smirnov test to define the type of distribution of the variables. Since the variables had a normal distribution, we used the parametric Student paired $t$ test. For categorical variables, we applied the $\chi^{2}$ test according to the expected frequency in the cell. When correlating continuous variables (spike rates vs fast oscillation rates), we computed the correlation coefficient. The level of significance was 0.05 . The data are presented as mean $\pm \mathrm{SD}$.

RESULTS Scalp EEG and clinical data. Duration of epilepsy in our 15 patients was $12.6 \pm 8.8$ years. The

Figure 2 Patient 11: Examples of artifacts and ripple oscillations
A

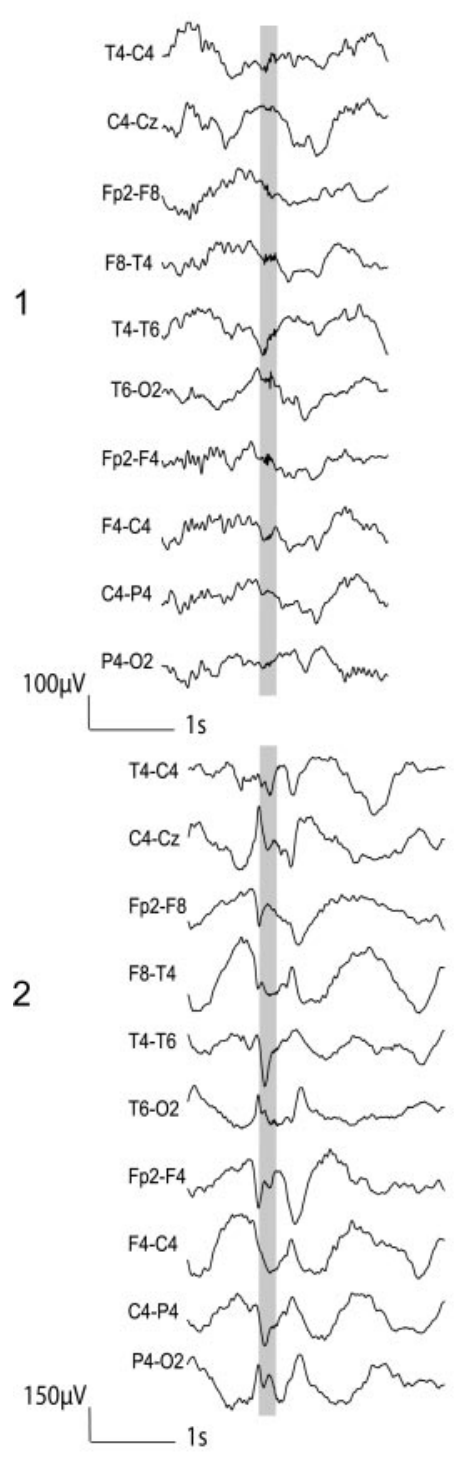

B

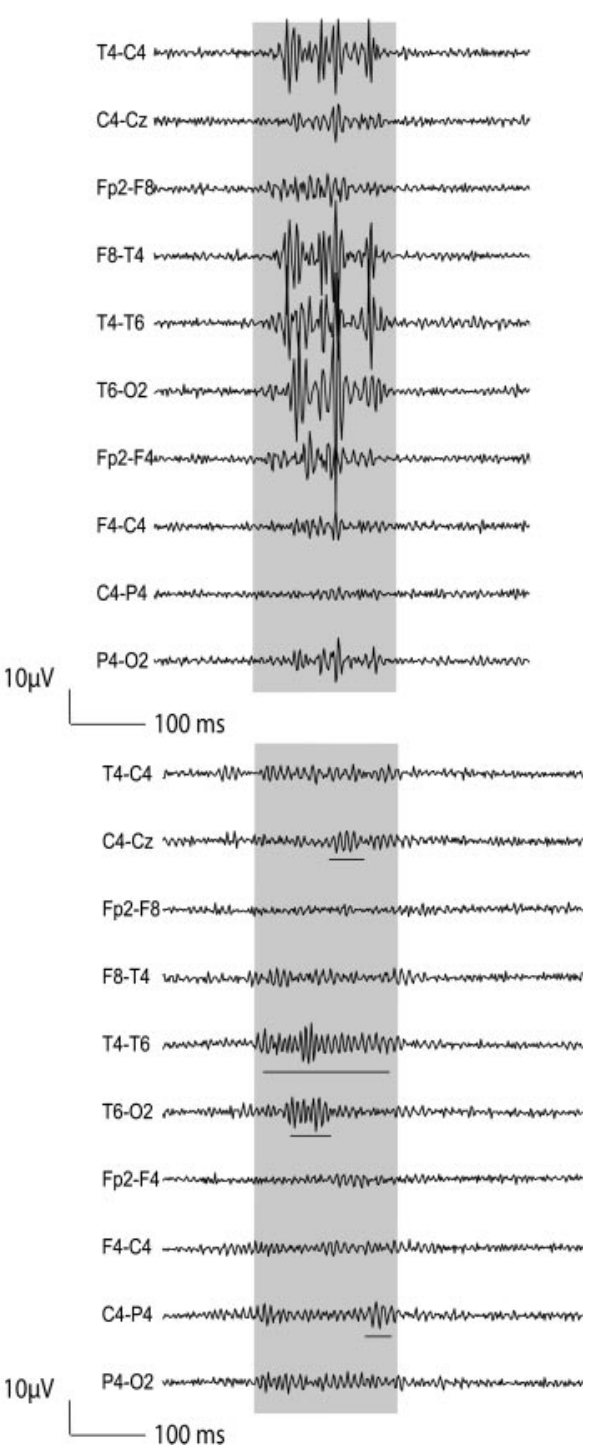

1) Short EMG bursts. 2) Ripples independent of epileptiform discharge. (A) Raw EEG. (B) EEG filtered with high-pass filter of $80 \mathrm{~Hz}$. The ripple oscillations are underlined. age at the first epileptic seizure was $18.5 \pm 11.6$ years. No lesion was detected in the MRI in 9/15 patients (60\%). During EEG telemetry all patients were taking their antiepileptic medication, which were tapered if necessary. Interictal spikes were detected in frontal, temporal, parietal, central, and occipital regions. We could identify a SOZ in $8 / 15$ patients (53.3\%); in 6/15 (40\%) the discharge at seizure onset was not localized or lateralized; one patient had no clinical seizure or ictal discharge. Table e-1 on the Neurology ${ }^{\circledR}$ Web site at www.neurology.org shows the clinical, neuroimaging, and scalp EEG data. Three patients underwent an intracerebral EEG study after noninvasive evaluation. Epilepsy surgery was indicated in 5 patients including 2 who had depth electrodes.

Artifacts. To minimize artifacts we studied only samples of non-REM sleep. After reviewing several records with combined filtered and unfiltered signals, we determined that almost all artifacts that could be confused with cerebral oscillations corresponded to short EMG bursts. Examples of artifacts and cerebral oscillations in the same patient are shown in figures 1-3. We developed a 2-step procedure to exclude these artifacts. The first step was during the identification of fast oscillations, when the channels were displayed with a $250 \mathrm{~mm} / \mathrm{s}$ time scale and filtered at 40 and $80 \mathrm{~Hz}$. We considered as artifacts the oscillations with irregular morphology, very high amplitude compared to the background, or great variations of amplitude and frequency during the train of oscillations. The waveform of nonartifactual fast oscillations was more rhythmic and regular in amplitude and frequency than was the irregular morphology of muscle activity. The second step was the elimination of oscillations marked in the first step if they were associated with muscle, movement, and electrode artifacts as identified on the EEG displayed with standard parameters $(30 \mathrm{~mm} / \mathrm{s})$.

Fast oscillations and interictal epileptiform discharges. Figures 4 and 5 show representative examples of gamma and ripples co-occurring with spikes and independently of spikes. Interestingly, we could detect some fast oscillations even in the raw EEG before filtering, when expanding the time scale. Figure e-1 also illustrates an interictal EEG viewed at successively expanded time scales without filtering and with a high pass filter at $5 \mathrm{~Hz}$ to remove only low frequencies, thus allowing a better appreciation of fast activity.

Gamma activity was recorded in all 15 patients and ripples in 12/15 (80\%). Gamma and ripples were recorded in all regions studied: frontal, temporal, parietal, central, and occipital. Considering all 
443 channels evaluated, the rates of occurrence were as follows: spikes $(1.63 \pm 2.04 / \mathrm{min})$, gamma $(0.36 \pm 0.83 / \mathrm{min})$, and ripples $(0.16 \pm 0.54 / \mathrm{min})$. Taking into account only the channels where these events were recorded, the rates were as follows: spikes $(1.93 \pm 2.08 / \mathrm{min} ; 374$ channels), gamma (0.65 \pm $1.03 / \mathrm{min} ; 242$ channels $)$, and ripples $(0.49 \pm 0.85 /$ min; 146 channels).

Increased rates of spikes were accompanied by increased rates of gamma $(p<0.001)$ and ripples $(p<$ $0.001)$. Gamma and ripples co-occurred with spikes in $77.5 \%$ and $63.4 \%$ of cases, respectively. Spikes co-occurred in $14.5 \%$ of cases with gamma and $6.6 \%$ with ripples. Gamma and ripples could be recorded without temporal overlap with spikes; however, in most cases they were recorded in spiking channels. In only 2 patients, in addition to being detected in spik-

\section{Figure 3 Patient 4: Examples of artifacts and gamma oscillations}

A

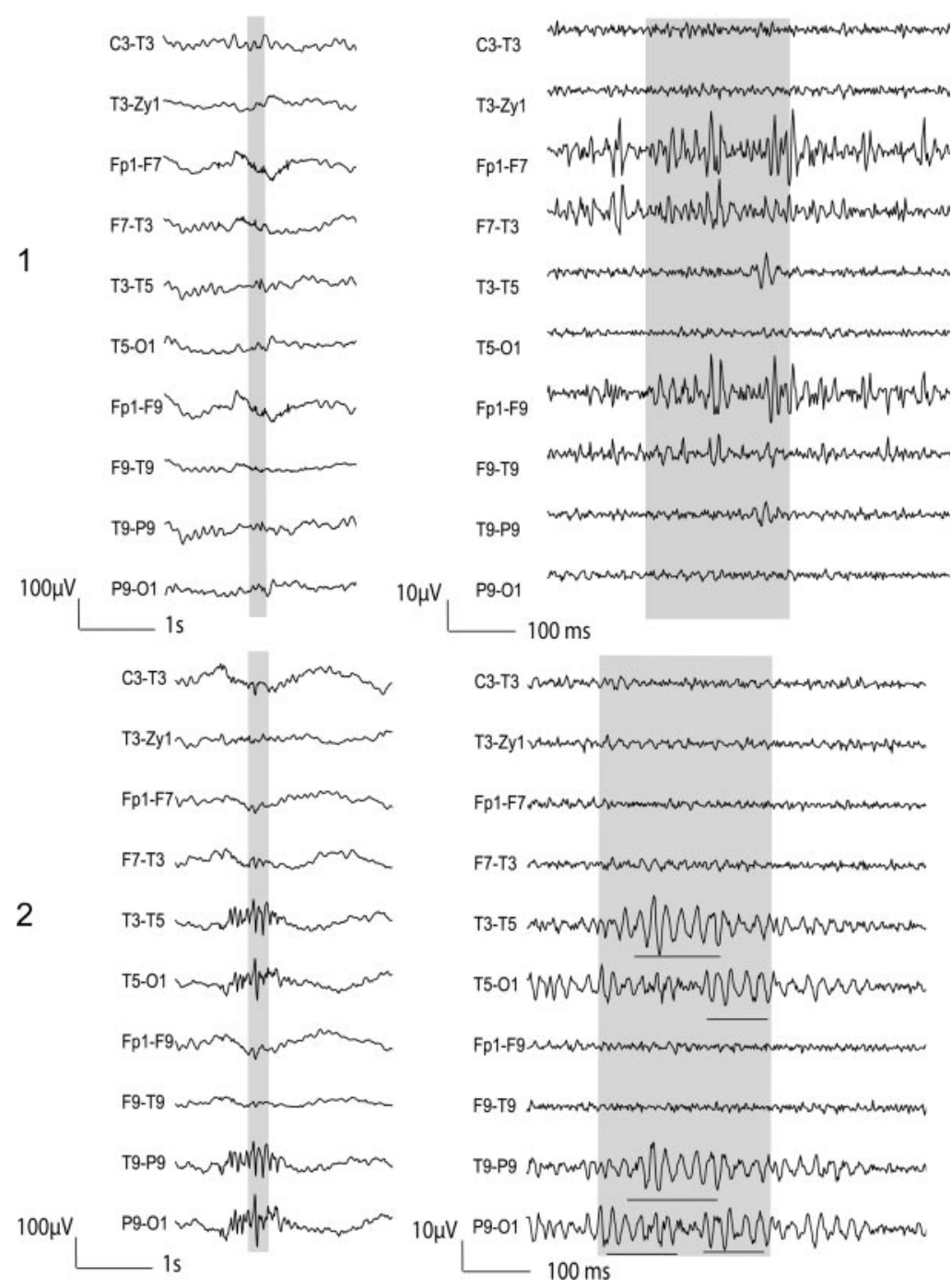

1) Short EMG bursts. 2) Gamma co-occurring with polyspikes. (A) Raw EEG. (B) EEG filtered with high-pass filter of $40 \mathrm{~Hz}$. The gamma oscillations are underlined. ing channels, fast activity was recorded in channels without spikes. In one of these patients, fast activity was seen over 2 nonspiking channels and in the other over one nonspiking channel; fast oscillations occurred in both at slower rates $(0.03 / \mathrm{min})$ than in spiking channels.

Fast oscillations and SOZ. In the 8 patients with a defined SOZ, 239 channels were studied: 195 nonSOZ channels and 44 SOZ channels. Among them, 181 spiking channels were detected. For all events, the proportion of channels inside the SOZ was consistently higher than outside the SOZ: spikes (44/44 [100\%] vs 137/195 [70.3\%], $\left.p<0.0001, \chi^{2}\right)$, gamma $(36 / 44$ [81.8\%] vs 65/195 [33.3\%], $p<$ $\left.0.0001, \chi^{2}\right)$, and ripples $(21 / 44$ [47.7\%] vs $22 / 195$ [11.3\%], $\left.p<0.0001, \chi^{2}\right)$. The number of channels in which spikes co-occurred with gamma $(31 / 44$ [70.5\%] vs 47/195 [24.1\%], $\left.p<0.0001, \chi^{2}\right)$ or ripples (16/44 [36.4\%] vs 15/195 [7.7\%], $p<0.0001$, $\chi^{2}$ ) was also significantly higher inside than outside the SOZ. Channels in which spikes co-occurred with fast oscillations were more reliable SOZ markers than channels in which spikes were not associated with fast oscillations (table e-2).

Additionally, for all events, the mean rates were higher inside than outside the SOZ: spikes $(2.64 \pm$ 1.70 vs $0.69 \pm 0.26, p=0.02$, paired $t$ test), gamma $(0.77 \pm 0.71$ vs $0.20 \pm 0.25, p=0.02)$, and ripples $(0.08 \pm 0.12$ vs $0.04 \pm 0.09, p=0.04)$. The sensitivity to identify the SOZ was $100 \%$ for spikes, $82 \%$ for gamma, and $48 \%$ for ripples; the specificity was $30 \%$ for spikes, $68 \%$ for gamma, and $89 \%$ for ripples; and the accuracy was $43 \%$ for spikes, $70 \%$ for gamma, and $81 \%$ for ripples.

DISCUSSION Several studies demonstrated the significance of oscillations in the 80 to $500 \mathrm{~Hz}$ range in the context of intracranial investigations in patients with focal epilepsy. ${ }^{1-4,6,7,21-23}$ These studies report that the HFO generator is very small: $1 \mathrm{~mm}^{3}$ using microelectrodes ${ }^{22}$ and in the range of 1 or a few $\mathrm{cm}^{3}$ using macroelectrodes. ${ }^{5}$ It has been shown that a much larger area of synchronized cortex (at least 6 to $9 \mathrm{~cm}^{2}$ ) is required for the corresponding activity to appear on the scalp. ${ }^{24}$ In this context, it is unlikely that the HFOs described in intracranial studies could be visible on the scalp. It is important to note that the skull and other tissues between brain and scalp do not attenuate the frequencies in the $80-500 \mathrm{~Hz}$ range more than the standard EEG frequencies below $40 \mathrm{~Hz},{ }^{25}$ despite what is sometimes written in EEG textbooks.

We embarked on this study in spite of this issue of generator size because several publications reported high-frequency activity recorded on the scalp. Inter- 
ictal fast oscillations in beta ${ }^{13}$ and gamma range ${ }^{15}$ were also recorded on scalp EEG in patients with generalized epilepsy, and in children with catastrophic epilepsies. ${ }^{14,16-19}$ Recently, ripples were recorded on scalp EEG in children with sleep-induced electrical status epilepticus. ${ }^{14}$

We confirmed that fast oscillations can be captured with scalp EEG. To our knowledge, interictal fast activity in the ripple and gamma band recorded over scalp EEG of adults with focal epilepsy has not so far been reported. Most importantly, this study shows that interictal fast oscillations have a significant relationship with interictal spikes and that ripples in particular may represent a better marker of the epileptogenic zone in focal epilepsy.

Why it is possible to record this high-frequency activity on the scalp, given what is known of its intracerebral generator? The explanation may come from the finding that oscillations above $200 \mathrm{~Hz}$ could, on

Figure 4 Examples of interictal gamma

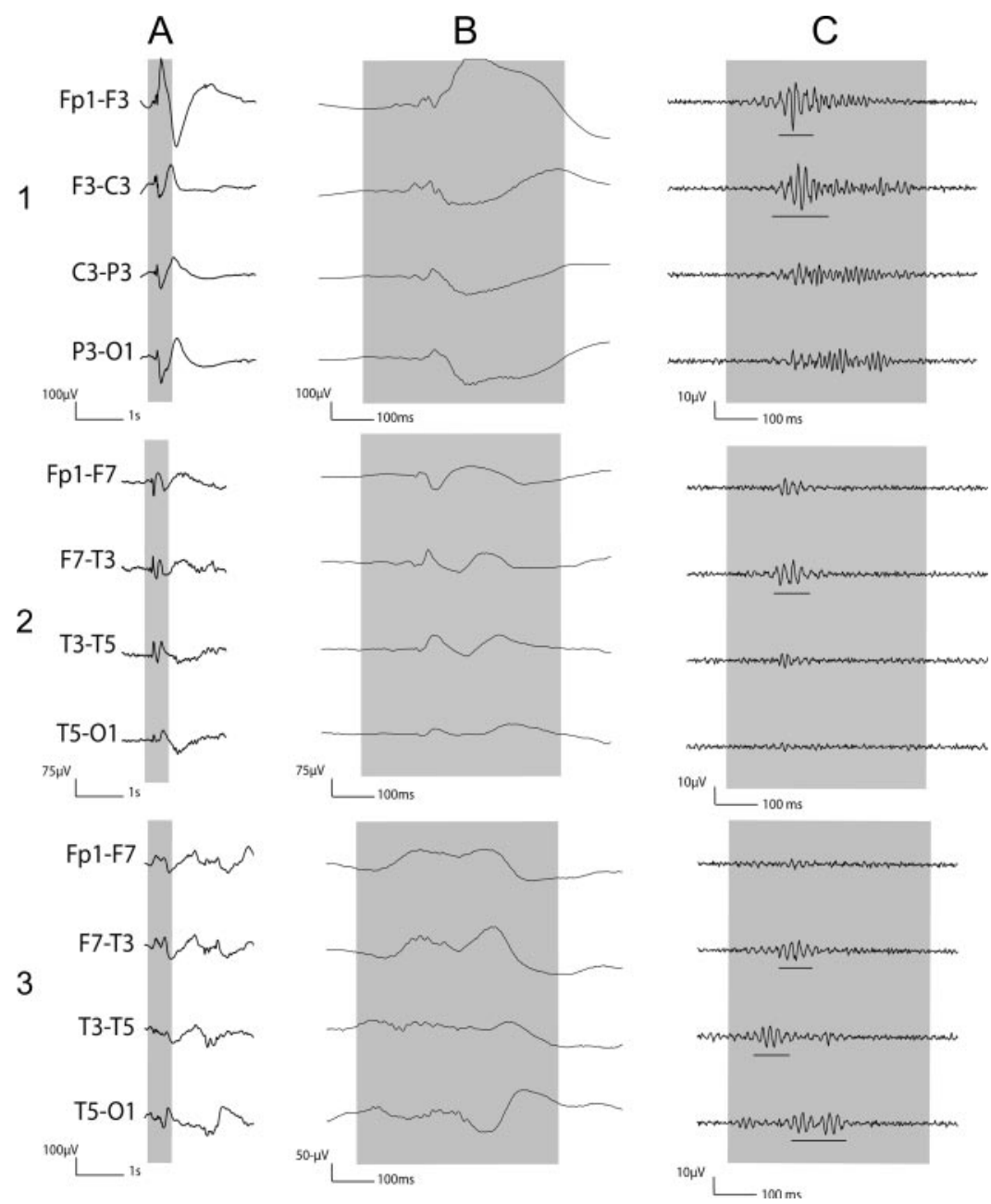

1) Gamma co-occurring with spike with oscillations visible during spike. 2) Gamma cooccurring with spike with oscillations not visible during spike, but visible after filtering. 3) Gamma independent of spike. (A) Raw EEG. (B) Raw EEG with expanded time. (C) EEG filtered with high-pass filter of $40 \mathrm{~Hz}$. Note different amplitude calibrations. rare occasions, occur simultaneously at electrodes separated by several centimeters. ${ }^{5}$ The rate of ripple activity that we report on the scalp $(0.49 / \mathrm{min})$ is much lower than the rate of $12.9 / \mathrm{min}$ reported intracerebrally. ${ }^{7}$ It would therefore be sufficient for 1 in every 26 intracerebral ripples on average to have a broader spatial extent, such that it could be recorded on the scalp.

Three of our patients underwent an intracerebral EEG investigation (data not shown). In these patients, HFOs were recorded in multiple neocortical contacts in electrodes located several centimeters apart. In all, there was good agreement between the intracerebral HFO generator regions and the field projection of these depth electrodes over the scalp regions where gamma and ripples were recorded. It is therefore possible that the occasional synchronization of HFOs across a large region results in a pattern visible on the scalp. Additionally, gamma activity is also present in intracranial recordings and may be synchronized over a broader region than activity above $80 \mathrm{~Hz}$. Future studies with simultaneous recording of depth and scalp electrodes are required to better address this question.

Although HFOs have often been recorded in deep mesial structures, it is unlikely that scalp fast oscillations are generated in these structures. Similarly, a scalp EEG seizure with temporal onset reflects seizure activity generated in temporal neocortex, even if the seizure may have originated in deep mesial structures. We had 2 patients with mesial temporal sclerosis and focal temporal scalp onset. In both we recorded fast oscillations in the SOZ. In these cases it is possible that the seizure started in mesial temporal structures, and propagated to the neocortex, where it became apparent on scalp EEG. In such a situation, the scalp fast oscillations would not reflect a true SOZ but only the seizure onset as apparent to scalp EEG. It is also possible that the seizures have a simultaneous mesial and neocortical onset, in which case scalp fast oscillations reflect a genuine SOZ. Unfortunately we cannot separate these 2 possibilities.

A low pass filter of $200 \mathrm{~Hz}$ and a sampling rate of $600 \mathrm{~Hz}$ were used, aiming to study gamma and ripple oscillations. We did not plan to record the fast ripple band $(200-500 \mathrm{~Hz})$ since activity in this band is much smaller in amplitude and more localized than ripples and gamma. Given the large number of patients in whom we found ripples, it appears that fast ripples should be investigated as well.

Discriminating biological artifacts from the EEG remains challenging for any neurophysiologic study. Muscle artifacts can even cause misinterpretation in intracranial EEG. ${ }^{26}$ When looking at high-frequency and low-amplitude activity in scalp EEG, it is critical 
to ensure that the EMG signal as a source for this fast activity has been eliminated. We employed the following strategies to reduce the risk of confusing artifacts with HFOs: 1) patients were studied during non-REM sleep (stages III and IV primarily); 2) before marking the fast activity we eliminated electrodes with malfunction or continuous artifacts; 3) in the oscillations, we identified morphologic features that appear typical of artifacts; and 4) after marking fast oscillations we reviewed the original EEG and excluded oscillations associated with artifacts identifiable in the standard record. We are therefore confident that the oscillations we report are of cerebral origin. Additionally, some fast activity could be detected in the EEG prior to filtering, reinforcing the view that these fast activities cannot result only from filtering sharp transients.

Figure $5 \quad$ Examples of interictal ripples
A
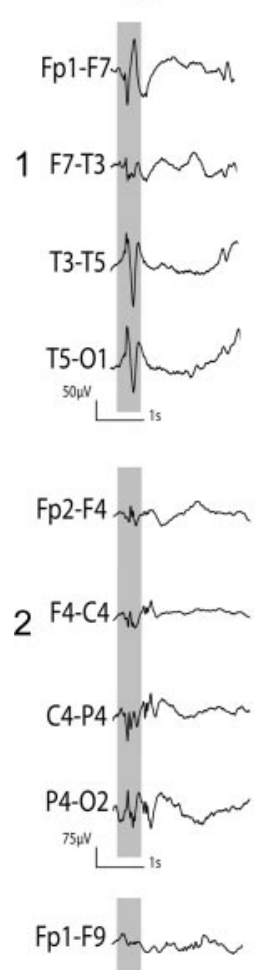

3

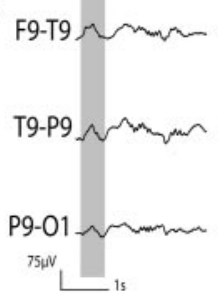

B
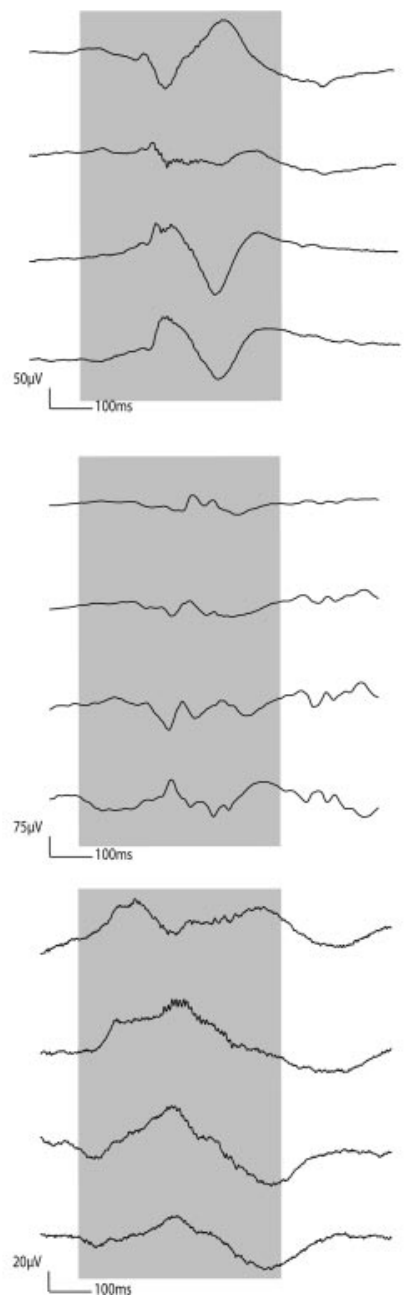
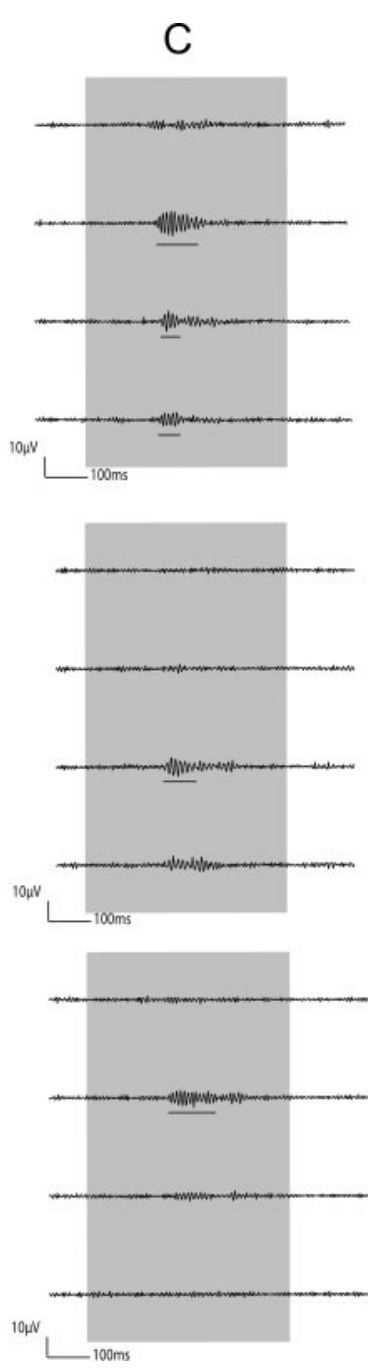

1) Ripples co-occurring with spike with oscillations visible during spike. 2) Ripples cooccurring with spike with oscillations not visible during spike, but visible after filtering. 3) Ripples independent of spike. (A) Raw EEG. (B) Raw EEG with expanded time. (C) EEG filtered with high-pass filter of $80 \mathrm{~Hz}$. Note different amplitude calibrations.
Under normal conditions, gamma oscillations have been observed in many cortical brain areas of different species and seem related to cognitive processes. ${ }^{27}$ There is clinical and experimental evidence that gamma oscillations play an important role in epilepsy. ${ }^{14-19,28-32}$ The generation of gamma oscillations appears related to the balance between tonic excitation (increased by the activation of NMDA receptor $\left.{ }^{31}\right)$ and tonic inhibition $\left(\mathrm{GABA}_{\mathrm{A}}\right.$ receptormediated inhibition) of interneurons, ${ }^{30,33}$ which is the cornerstone of seizure generation.

Human intracranial recordings showed that immediately before or at the onset of an epileptic seizure there is often an increase in the amplitude of the $40-120 \mathrm{~Hz}$ range ${ }^{34}$ and the presence of localized high-frequency activity in the SOZ, between 20 and $80 \mathrm{~Hz}$, was associated with a good postoperative outcome. ${ }^{35}$ Furthermore, magnetoencephalography detects spike-locked and spike-independent gamma oscillations which correctly identified the SOZ in 5/6 patients who underwent presurgical workup with simultaneous magnetoencephalography and intracranial recordings. ${ }^{36}$ These studies support the potential importance of scalp-recorded gamma and ripple activity in focal epilepsy, although their relation to the SOZ has not been established, particularly with respect to the ripple.

We demonstrated that the rates of fast oscillations and the number of channels with gamma and ripples are higher inside the SOZ, and spikes co-occurring with fast oscillations were a more reliable marker of the SOZ than spikes not associated with fast oscillations; although less sensitive these events are more specific and accurate than spikes to delineate the SOZ. We feel that both frequency bands have a role in epileptic activity and should continue to be studied. The data have to be interpreted with caution since we have not related yet the presence of scalp fast activity to surgical outcome, as done for intracranial HFOs. ${ }^{10}$

A potential neurophysiologic method is emerging with the use of interictal fast oscillations as a scalp marker of the SOZ. The possibility to detect fast oscillations noninvasively may considerably extend their use in the presurgical evaluation of epileptic patients and the investigation of epileptic disorders.

\section{AUTHOR CONTRIBUTIONS}

Dr. Andrade-Valença: drafting/revising the manuscript, study concept or design, analysis or interpretation of data, acquisition of data, statistical analysis. Dr. Dubeau: drafting/revising the manuscript, study concept or design, analysis or interpretation of data, study supervision. Dr. Mari: analysis or interpretation of data, acquisition of data. R. Zelmann: study concept or design, analysis or interpretation of data, signal processing of data. Dr. Gotman: drafting/revising the manuscript, study concept or design, analysis or interpretation of data, study supervision, obtaining funding. 


\section{ACKNOWLEDGMENT}

The authors thank Lorraine Allard and Nicole Drouin for technical EEG support and Natalja Zazubovitz for help with the figures (Montreal Neurological Institute).

\section{DISCLOSURE}

Dr. Andrade-Valenca received scholarship support from the Canadian Institutes of Health Research. Dr. Dubeau serves as an Associate Editor for Epileptic Disorders and received research support from the Canadian Institutes of Health Research. Dr. Mari received scholarship support from the Canadian Institutes of Health Research. R. Zelmann received scholarship support from the National Science and Engineering Research Council, Canada. Dr. Gotman has received funding for travel and research support from the Canadian Institutes of Health Research; serves on the editorial boards of Epilepsia and the Journal of Clinical Neurophysiology; and serves as a consultant for (and is former CEO of) Stellate and Lacerta Research, in both of which he is a major stockholder.

Received September 17, 2010. Accepted in final form February 7, 2011.

\section{REFERENCES}

1. Bragin A, Engel J Jr, Wilson CL, Fried I, Mathern GW. Hippocampal and entorhinal cortex high-frequency oscillations $(100-500 \mathrm{~Hz})$ in human epileptic brain and in kainic acid-treated rats with chronic seizures. Epilepsia 1999;40:127-137.

2. Bragin A, Engel J Jr, Wilson CL, Vizentin E, Mathern GW. Electrophysiologic analysis of a chronic seizure model after unilateral hippocampal KA injection. Epilepsia 1999;40:1210-1221.

3. Staba RJ, Wilson CL, Bragin A, Fried I, Engel J Jr. Quantitative analysis of high-frequency oscillations (80-500 $\mathrm{Hz}$ ) recorded in human epileptic hippocampus and entorhinal cortex. J Neurophysiol 2002;88:1743-1752.

4. Jirsch JD, Urrestarazu E, LeVan P, Olivier A, Dubeau F, Gotman J. High-frequency oscillations during human focal seizures. Brain 2006;129:1593-1608.

5. Crepon B, Navarro V, Hasboun D, et al. Mapping interictal oscillations greater than $200 \mathrm{~Hz}$ recorded with intracranial macroelectrodes in human epilepsy. Brain 2010;133: 33-45.

6. Urrestarazu E, Chander R, Dubeau F, Gotman J. Interictal high-frequency oscillations $(100-500 \mathrm{~Hz})$ in the intracerebral EEG of epileptic patients. Brain 2007;130:23542366.

7. Jacobs J, LeVan P, Chander R, Hall J, Dubeau F, Gotman J. Interictal high-frequency oscillations $(80-500 \mathrm{~Hz})$ are an indicator of seizure onset areas independent of spikes in the human epileptic brain. Epilepsia 2008;49:1893-1907.

8. Worrell GA, Gardner AB, Stead SM, et al. High-frequency oscillations in human temporal lobe: simultaneous microwire and clinical macroelectrode recordings. Brain 2008;131:928-937.

9. Zijlmans M, Jacobs J, Zelmann R, Dubeau F, Gotman J. High-frequency oscillations mirror disease activity in patients with epilepsy. Neurology 2009;72:979-986.

10. Jacobs J, Zijlmans M, Zelmann R, et al. High-frequency electroencephalographic oscillations correlate with outcome of epilepsy surgery. Ann Neurol 2010;67:209-220.

11. Nealis JG, Duffy FH. Paroxysmal beta activity in the pediatric electroencephalogram. Ann Neurol 1978;4:112-116.

12. Rodin E, Smid N, Mason K. The grand mal pattern of Gibbs, Gibbs and Lennox. Electroencephalogr Clin Neurophysiol 1976;40:401-406.
13. Halasz P, Janszky J, Barcs G, Szucs A. Generalised paroxysmal fast activity (GPFA) is not always a sign of malignant epileptic encephalopathy. Seizure 2004;13:270-276.

14. Kobayashi K, Watanabe Y, Inoue T, Oka M, Yoshinaga H, Ohtsuka Y. Scalp-recorded high-frequency oscillations in childhood sleep-induced electrical status epilepticus. Epilepsia 2010;51:2190-2194.

15. Willoughby JO, Fitzgibbon SP, Pope KJ, et al. Persistent abnormality detected in the non-ictal electroencephalogram in primary generalised epilepsy. J Neurol Neurosurg Psychiatry 2003;74:51-55.

16. Wu JY, Koh S, Sankar R, Mathern GW. Paroxysmal fast activity: an interictal scalp EEG marker of epileptogenesis in children. Epilepsy Res 2008;82:99-106.

17. Yamazaki M, Chan D, Tovar-Spinoza Z, et al. Interictal epileptogenic fast oscillations on neonatal and infantile EEGs in hemimegalencephaly. Epilepsy Res 2009;83: 198-206.

18. Kobayashi K, Oka M, Akiyama T, et al. Very fast rhythmic activity on scalp EEG associated with epileptic spasms. Epilepsia 2004;45:488-496.

19. Inoue T, Kobayashi K, Oka M, Yoshinaga H, Ohtsuka Y. Spectral characteristics of EEG gamma rhythms associated with epileptic spasms. Brain Dev 2008;30:321-328.

20. Bagshaw AP, Jacobs J, LeVan P, Dubeau F, Gotman J. Effect of sleep stage on interictal high-frequency oscillations recorded from depth macroelectrodes in patients with focal epilepsy. Epilepsia 2009;50:617-628.

21. Staba RJ, Wilson CL, Bragin A, Jhung D, Fried I, Engel J Jr. High-frequency oscillations recorded in human medial temporal lobe during sleep. Ann Neurol 2004;56:108115.

22. Bragin A, Engel J Jr, Wilson CL, Fried I, Buzsaki G. Highfrequency oscillations in human brain. Hippocampus 1999;9:137-142.

23. Urrestarazu E, Jirsch JD, LeVan P, et al. High-frequency intracerebral EEG activity $(100-500 \mathrm{~Hz})$ following interictal spikes. Epilepsia 2006;47:1465-1476.

24. Tao JX, Baldwin M, Hawes-Ebersole S, Ebersole JS. Cortical substrates of scalp EEG epileptiform discharges. J Clin Neurophysiol 2007;24:96-100.

25. Oostendorp TF, Delbeke J, Stegeman DF. The conductivity of the human skull: results of in vivo and in vitro measurements. IEEE transactions on bio-medical engineering 2000;47:1487-1492.

26. Otsubo H, Ochi A, Imai K, et al. High-frequency oscillations of ictal muscle activity and epileptogenic discharges on intracranial EEG in a temporal lobe epilepsy patient. Clin Neurophysiol 2008;119:862-868.

27. Herrmann CS, Frund I, Lenz D. Human gamma-band activity: a review on cognitive and behavioral correlates and network models. Neurosci Biobehav Rev 2010;34: 981-992.

28. Medvedev A, Mackenzie L, Hiscock JJ, Willoughby JO. Kainic acid induces distinct types of epileptiform discharge with differential involvement of hippocampus and neocortex. Brain Res Bull 2000;52:89-98.

29. Medvedev AV. Epileptiform spikes desynchronize and diminish fast (gamma) activity of the brain: an "antibinding” mechanism? Brain Res Bull 2002;58:115-128.

30. Kohling R, Vreugdenhil M, Bracci E, Jefferys JG. Ictal epileptiform activity is facilitated by hippocampal GABAA receptor-mediated oscillations. J Neurosci 2000;20:68206829. 
31. Traub RD, Pais I, Bibbig A, et al. Transient depression of excitatory synapses on interneurons contributes to epileptiform bursts during gamma oscillations in the mouse hippocampal slice. J Neurophysiol 2005;94:1225-1235.

32. Herrmann CS, Demiralp T. Human EEG gamma oscillations in neuropsychiatric disorders. Clin Neurophysiol 2005;116:2719-2733.

33. Khazipov R, Holmes GL. Synchronization of kainate-induced epileptic activity via GABAergic inhibition in the superfused rat hippocampus in vivo. J Neurosci 2003;23:5337-5341.
34. Fisher RS, Webber WR, Lesser RP, Arroyo S, Uematsu S. High-frequency EEG activity at the start of seizures. J Clin Neurophysiol 1992;9:441-448.

35. Alarcon G, Binnie CD, Elwes RD, Polkey CE. Power spectrum and intracranial EEG patterns at seizure onset in partial epilepsy. Electroencephalogr Clin Neurophysiol 1995;94:326-337.

36. Rampp S, Kaltenhauser M, Weigel D, et al. MEG correlates of epileptic high gamma oscillations in invasive EEG. Epilepsia 2010;51:1638-1642.

\section{$+4+$ CELEBRATING 60 YEARS

\section{THE SHORTHAND PUBLICATIONS OF SIR WILLIAM RICHARD GOWERS}

Kenneth L. Tyler, Dorothy Roberts, and H. Richard Tyler

Neurology 2000;55:289-293

Objective: To examine the shorthand publications of Sir William Richard Gowers. Background: Gowers developed an almost obsessional interest in Pitman shorthand. During the later part of his active career (1894-1910), the bulk of his professional writing, comprising nearly 100 articles, was printed entirely in Pitman shorthand in the Phonographic Record of Clinical Teaching and Medical Science. The obscurity and rarity of this periodical, and the increasingly arcane nature of Pitman shorthand, has left the bulk of these articles "buried in obscurity and locked up in code" (M. Critchley, 1949). Design/Methods: A complete set of Gowers' shorthand publications as listed in his standard bibliography was compiled. Transcription of the Pitman shorthand outlines was performed by a qualified Pitman shorthand transcriber and verified using the contemporaneous Phonographic Outlines of Medical Terms (1902) as an authoritative guide. Results: The first transcription of Gowers' shorthand publications has now been completed. The history of Gowers' interest in shorthand and his efforts to proselytize the medical profession is reviewed. Selected excerpts are presented from his shorthand articles, which include papers devoted to problems in practical diagnosis, notes on clinical teaching, and the shorthand transcriptions of his lectures at Queen Square and at University College Hospital on such diverse subjects as myelitis, neurosyphilis, polio, muscular dystrophy, tumors, vascular disease, epilepsy, and the nervous system in old age. Conclusions: The previously unpublished transcriptions of his shorthand articles represent a major and previously inaccessible part of Gowers' neurologic opus. These articles exemplify Gowers as a practicing neurologist and teacher, and significantly expand our insights into one of neurology's most significant and influential figures.

Free Access to this article at www.neurology.org/content/55/2/289

Comment from Robert A. Gross, MD, PhD, FAAN, Editor-in-Chief: An analysis of little-seen (or read) primary documentation. In another article (Neurology 1996;46:1467-1469; Historical Abstract reprinted in July 12, 2011, issue), Sacks mentions the short-hand notation in his exploration of Gowers' memory. 